\title{
Comparison of technical and physical activities between 8 vs. 8 and 11 vs. 11 games in young Korean soccer players
}

\author{
Seong Hwan $\mathrm{Oh}^{1}$, Chang Hwa Joo ${ }^{2 *}$ \\ 'Department of Technical Research, Korea Football Association, Paju, Korea \\ ${ }^{2}$ Department of Football Science, Honam University, Gwangju, Korea
}

The aims of this study were to examine the differences in technical aspects and physical demands between small-size games (SSG; 8 vs. 8) and regular-size games (RSG; 11 vs. 11) in young Korean soccer players. Seventy-nine young soccer players from 6 teams (U-12) volunteered to participate in the study. The players completed 4 games (2 SSG, $62 \times 51 \mathrm{~m}$, and $2 \mathrm{RSG}, 80 \times 54 \mathrm{~m}$ ) in 2 days. Each game was filmed to evaluate technical actions. Physical demand variables were measured using global positioning system technology. SSG showed significantly greater numbers of technical plays among 17 variables when compared to RSG $(P<0.05)$. The players covered significantly greater total distance during low-, moderate-, and high-speed running and sprinting in SSG than in RSG $(P<0.05)$. Higher numbers of high-intensity activities (repeated high-intensity efforts, explosive efforts, decelerations, accelerations, and sprinting) were observed in SSG compared to RSG $(P<0.05)$. Mean heart rate was also higher in SSG than in RSG $(P<0.05)$. Despite the greater physical demands during SSG, the exercise intensity was similar to that reported in previous studies. Therefore, the SSG format applied in the present study can be a suitable official game format for Korean young soccer players, resulting in significantly greater exposure to technical plays without excessive physical demands.

Keywords: Small-sided soccer games, Young players, Match analysis, Technical variables, Physical demands

\section{INTRODUCTION}

Top level soccer players cover about $11 \mathrm{~km}$ and perform 17-35 sprints during a 90-min soccer game (Bradley et al., 2014; Di Salvo et al., 2010). A high fitness level is required to maintain technical performance such as accuracy of passing and shooting by the end of a soccer game. In order to improve the performance of soccer players, various training methods have been developed and used over the last few decades. The most effective training methods used to improve performance incorporate conditions similar those in an actual soccer game (Aguiar et al., 2012). Small-size games (SSG) are widely used by soccer coaches to improve physical fitness and technique in soccer teams, ranging from youth to senior levels.

Exercise intensity and technical performance when players train with the ball can be influenced by the number of players involved, the size and the shape of the pitch, the duration of exercise, the ratio of exercise to rest, the rules of the game, coach encouragement, the availability of balls, the way points are scored, and the pitch conditions (Aguiar et al., 2012; Bansbo, 1994; Hill-Haas et al., 2009). Exercise intensity in SSG may also be affected by these factors. For example, Abrantes et al. (2012) reported that the lower number of players with smaller area increased exercise intensity in SSG. On the other hand, soccer players perform more technical skills such as shooting and dribbling in 3 vs. 3 compared with 6 vs. 6 games (Katis and Kellis, 2009).

The purpose of training programs for childhood players (under 12 years) should be to enhance the performance of techniques rather than physical fitness. In the childhood period, it is necessary to organize training in the form of exercise that can improve coordination between the nervous system and muscles; this is closely related to development of soccer skills, rather than training
${ }^{*}$ Corresponding author: Chang Hwa Joo (D) https://orcid.org/0000-0002-7429-3104 Department of Football Science, Honam University, 417 Eodeung-daero, Gwangsan-gu, Gwangju 62399, Korea

Tel: +82-62-940-3602, Fax: +82-62-940-3741, E-mail: footballer@honam.ac.kr Received: January 1, 2018 / Accepted: January 30, 2018
This is an Open Access article distributed under the terms of the Creative Commons Attribution Non-Commercial License (http://creativecommons.org/licenses/by-nc/4.0/) which permits unrestricted non-commercial use, distribution, and reproduction in any medium, provided the original work is properly cited. 
for muscle strength and cardiopulmonary endurance (Reilly et al., 2000). For this reason, exercise with the ball is important in this period. Bangsbo (2007) reported that there was no difference in physical fitness at the senior level between groups focused on technique-based training with the ball and those performing traditional physical training during childhood. Indeed, SSG training is used to improve skills for U-12 soccer players, and 7 vs. 7 and/ or 8 vs. 8 SSG have been applied in official games in European countries playing a high level of soccer.

There are many studies on the physical and technical effects of SSG in youth and senior soccer players (Casamichana and Castellano, 2010; Castellano et al., 2013; Kelly and Drust, 2009), whereas limited data have been collected on the impact of SSG in young players. Capranica et al. (2001) compared match performance and physiological load during regular-size games (RSG) in 11 -year-old soccer players (11 vs. 11 on a regular-size pitch, $100 \times 65 \mathrm{~m}$ ) and SSG (7 vs. 7 on a smaller pitch, $60 \times 40 \mathrm{~m}$ ). The results indicated that more passes and fewer tackles occurred in SSG, while physiological load was similar between the match formats. These findings are supported by recent findings indicating that young Korean soccer players performed significantly more technical plays during SSG (8 vs. 8) than during RSG (Joo et al., 2016).

The previous studies had several limitations, including the failure to use a control condition in which teams play with the same game formats (SSG and RSG). Differences in physical and technical levels of opponent teams between game formats can affect the results of physical activities and performance of the skills. For example, a higher level team can apply more technique (i.e., passing and shooting) than a lower level team during a game because the former possesses the ball more times. Therefore, a more controlled methodological approach is needed to improve reliability of research data to clarify differences in physical activities and technical performance in young players according to SSG and RSG game formats.

\section{MATERIALS AND METHODS}

\section{Subjects}

Seventy-nine young soccer players from 6 teams (U-12) volunteered to participate in the study. All players were members of elite soccer teams registered with the Korea Football Association (KFA) and the coaches were certified by the Asian Football Confederation. The players had $>2$ years of soccer training experience with $\geq 5$ sessions per week. The players played continuously for
25 min on grass without substitution or a half-time break. The field players who did not play in both formats and goalkeepers were excluded from video analysis after the games. All experimental protocols and related procedures were approved by the ethics committee of Honam University (approval number: 1041223201405-HR-007-01).

\section{Experimental procedures}

The 6 teams played 24 games in 2 days. A team played two game formats with the same opposing team on the first day, with at least a 2-hr recovery period between games, and with another opponent on the next day (counterbalanced, randomized crossover design). The players were advised to drink water during the experimental period. Two game formats were used to provide an equal area per player $\left(197 \mathrm{~m}^{2}\right)$. SSG format consisted of 8 players per side, playing on a small-size field $(62 \times 51 \mathrm{~m})$, and RSG format consisted of 11 players per side, playing on a regular-size field $(80 \times 54 \mathrm{~m})$.

All matches were performed on natural turf and modified rules based on KFA official soccer rules were strictly applied: (a) The players were not allowed to shoot directly at kick-off. (b) The players could not pass the ball over the halfway line at goal kick. (c) The goalkeeper could not kick or throw the ball over the halfway line during in-play situations (an indirect free kick was given for a violation of these rules). (d) Many balls were located around the court to ensure fast return to play. (e) Verbal support from the coaches was not allowed during the matches to control for confounding factors between game formats.

\section{Match analyses}

The games were filmed using a video camera to analyze technical actions. Technical factors were analyzed by a specialist (Visual Sports) in soccer game analysis with more than 5 years of experience. In this study, one variable was assessed for the number of ball touches, successful dribbles, crosses, cutbacks, and aerial duels during matches. Twelve pass variables were assessed: all, the number of passes in defense, offense, and 1/3 area (offensive attacks in the 1/3 area of the field that contains the opponent team's goal), and successful passes in each area, 1-touch passes (nonstop passes), forward passes (pass range, $315^{\circ}$ to $\left.45^{\circ}\right)$, short-distance passes $(\leq 10 \mathrm{~m})$, middle-distance passes $(10 \mathrm{~m}$ to $<25 \mathrm{~m})$ and long-distance passes $(\geq 25 \mathrm{~m})$. Lastly, 3 shot variables were assessed: all goal shots (total number of attempted goal shots), shots in penalty area, and valid goal shots.

Physical demands were assessed based on the work-rate profiles observed during a match. The data were collected at $10 \mathrm{~Hz}$ 
during the games using a minimaxX global positioning system (GPS) unit (Team 2.5, Catapult Innovations, Melbourne, Australia). The GPS unit included triaxial accelerometers and gyroscopes sampling at $100 \mathrm{~Hz}$ to provide greater accuracy of measurement for speed, acceleration, deceleration, and repeated high- intensity efforts. This system has previously been shown to yield valid and reliable data (Rampinini et al., 2015). The work rates were categorized by the degree of movement intensity. The movement intensities were categorized according to 6 movements: walking (0.0-6.9 km/hr), jogging (7.0-9.9 km/hr), low-speed running (LSR, 10.0-12.9 km/hr), moderate-speed running (MSR, 13.0$15.9 \mathrm{~km} / \mathrm{hr}$ ), high-speed running (HSR, 16.0-17.9 km/hr), and sprinting ( $\geq 18.0 \mathrm{~km} / \mathrm{hr}$ ) (Adade et al., 2014; Hill-Haas et al., 2009). Six additional variables were assessed to elucidate the physical demands on players: total distance covered (m), number of sprints, acceleration $\left(\geq 1 \mathrm{~m} / \mathrm{sec}^{2}\right)$, deceleration $\left(\leq-1.0 \mathrm{~m} / \mathrm{sec}^{2}\right)$, explosive effort, and repeated high intensity efforts. Explosive effort refers to the sum of high-intensity activities composed of running $(\geq 2.5$ $\mathrm{m} / \mathrm{sec}^{2}$ ) and jumping $(>20 \mathrm{~cm})$. Repeated high-intensity efforts were defined as $\geq 3$ high-speed runs ( $\geq 14.4 \mathrm{~km} / \mathrm{hr}$ ), rapid acceleration $\left(\geq 2.79 \mathrm{~m} / \mathrm{sec}^{2}\right)$, rapid deceleration $\left(\leq-2.79 \mathrm{~m} / \mathrm{sec}^{2}\right)$, and explosive effort with $<21$ sec of recovery between efforts. Heart rate (HR) was monitored every $5 \mathrm{sec}$ via short-range radio telemetry during the games. Mean HR was calculated for each game period.

\section{Statistical analyses}

Differences in technical and physical variables between the game formats were evaluated using a paired $t$-test. The significance level was 0.05 . Data are presented as mean \pm standard deviation. The data were analyzed using IBM SPSS ver. 18.0 (IBM Co., Armonk, NY, USA).

\section{RESULTS}

There were significant differences in several technical play variables between SSG and RSG (Fig. 1). A significantly greater number of total ball touches $(27.2 \pm 8.2$ vs. $20.1 \pm 79)$, all passes $(14.1 \pm 5.4$ vs. $9.2 \pm 5.1)$, and successful passes $(9.9 \pm 4.3$ vs. $5.9 \pm$

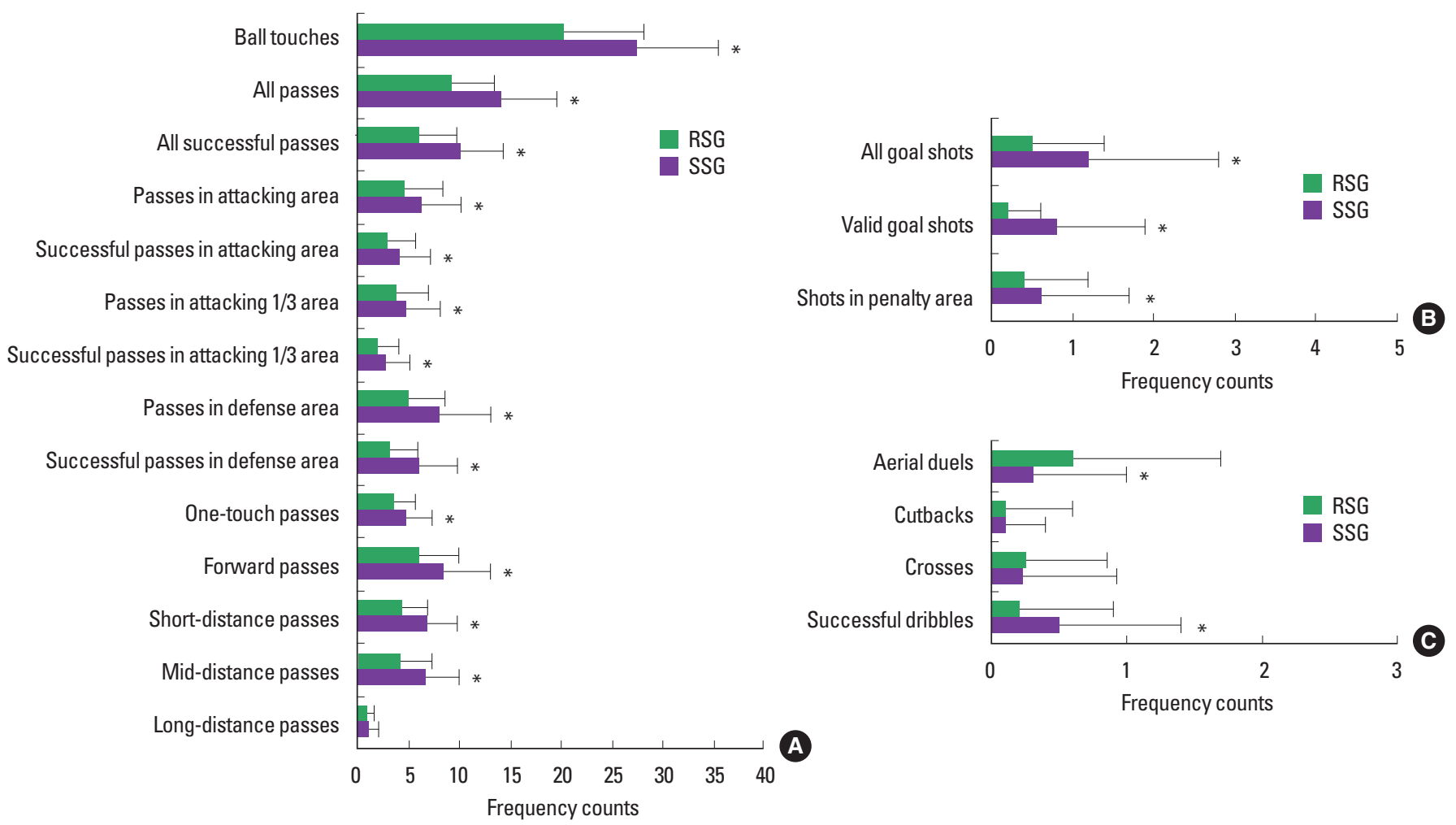

Fig. 1. Comparative analyses of the technical play variables of the players assessed during the SSG and RSG. (A) Differences in technical plays of the players (ball touches \& passes). (B) Differences in technical plays of the players (shots). (C) Differences in technical plays of the players (aerial duels, cut-backs, crosses, dribbles). The bars express the values calculated in mean \pm standard deviation. SSG, 8-a-side game with a small pitch dimension; RSG, 11-a-side game with a regular pitch dimension. ${ }^{*} P<0.05$, comparison between SSG and RSG. 
3.8); passes (6.1 \pm 4.1 vs. $4.4 \pm 3.9)$ and successful passes (4.1 \pm 3.0 vs. $2.8 \pm 2.9)$ in the attacking area; passes ( $4.6 \pm 3.6$ vs. $3.6 \pm 3.3)$ and successful passes $(2.7 \pm 2.4$ vs. $1.9 \pm 2.2)$ in the attacking $1 / 3$ area; passes ( $8.0 \pm 5.0$ vs. $4.8 \pm 3.8)$ and successful passes $(5.8 \pm 4.0$ vs. $3.1 \pm 2.7)$ in the defense area; 1 -touch passes $(4.6 \pm 2.7$ vs. $3.4 \pm$ 2.3); forward passes ( $8.4 \pm 4.6$ vs. $5.9 \pm 4.0)$; and short- $(6.7 \pm 3.0$ vs. $4.3 \pm 2.5)$ and mid-distance passes $(6.4 \pm 3.5$ vs. $4.1 \pm 3.1)$ were observed in SSG than in RSG $(P<0.05)$ (Fig. $1 \mathrm{~A})$. A significantly higher number of all goal shots ( $1.2 \pm 1.6$ vs. $0.5 \pm 0.9)$, valid goal shots $(0.8 \pm 1.1$ vs. $0.2 \pm 0.4)$, shots in penalty area $(0.6 \pm 1.1$ vs. $0.4 \pm 0.8)$ and successful dribbles $(0.5 \pm 0.9$ vs. $0.2 \pm 0.7)$ were observed in SSG than in RSG $(P<0.05)$ (Fig. 1B, C). The number of aerial duels was greater in RSG $(0.6 \pm 1.1)$ than in SSG $(0.3 \pm$ $0.7, P<0.05)$.

The physical demand variables are shown in Fig. 2. A signifi- cantly greater total distance $(2,443 \pm 268 \mathrm{~m}$ vs. $2,329 \pm 264 \mathrm{~m})$ was covered in LSR ( $407 \pm 102 \mathrm{~m}$ vs. $374 \pm 107 \mathrm{~m})$, MSR (227 \pm $61 \mathrm{~m}$ vs. $203 \pm 72 \mathrm{~m})$, HSR $(93 \pm 35 \mathrm{~m}$ vs. $74 \pm 40 \mathrm{~m})$, and sprinting $(78 \pm 41 \mathrm{~m}$ vs. $55 \pm 49 \mathrm{~m})$ in SSG compared to RSG $(P<0.05)$. However, a significantly greater distance was covered by walking in RSG $(1,067 \pm 73 \mathrm{~m})$ than in SSG $(1,102 \pm 80 \mathrm{~m}, P<0.05)$ (Fig. $2 A)$. The players performed a significantly higher number of repeated high-intensity efforts ( $9.9 \pm 4.6$ vs. $7.4 \pm 4.5)$, explosive efforts $(30.5 \pm 24.2$ vs. $24.4 \pm 9.6)$, decelerations ( $27.1 \pm 7.0$ vs. $23.9 \pm 7.7$ ), accelerations ( $41.6 \pm 10.6$ vs. $35.3 \pm 9.7$ ), and sprints $(8.3 \pm 4.1$ vs. $5.6 \pm 4.1)$ during SSG compared to RSG $(P<0.05)$ (Fig. 2B). Similarly, mean HR during the games was higher in SSG $(168 \pm 13$ beats/min) than in RSG $(163 \pm 15$ beats/min) $(P<0.05)$ (Fig. 2C).

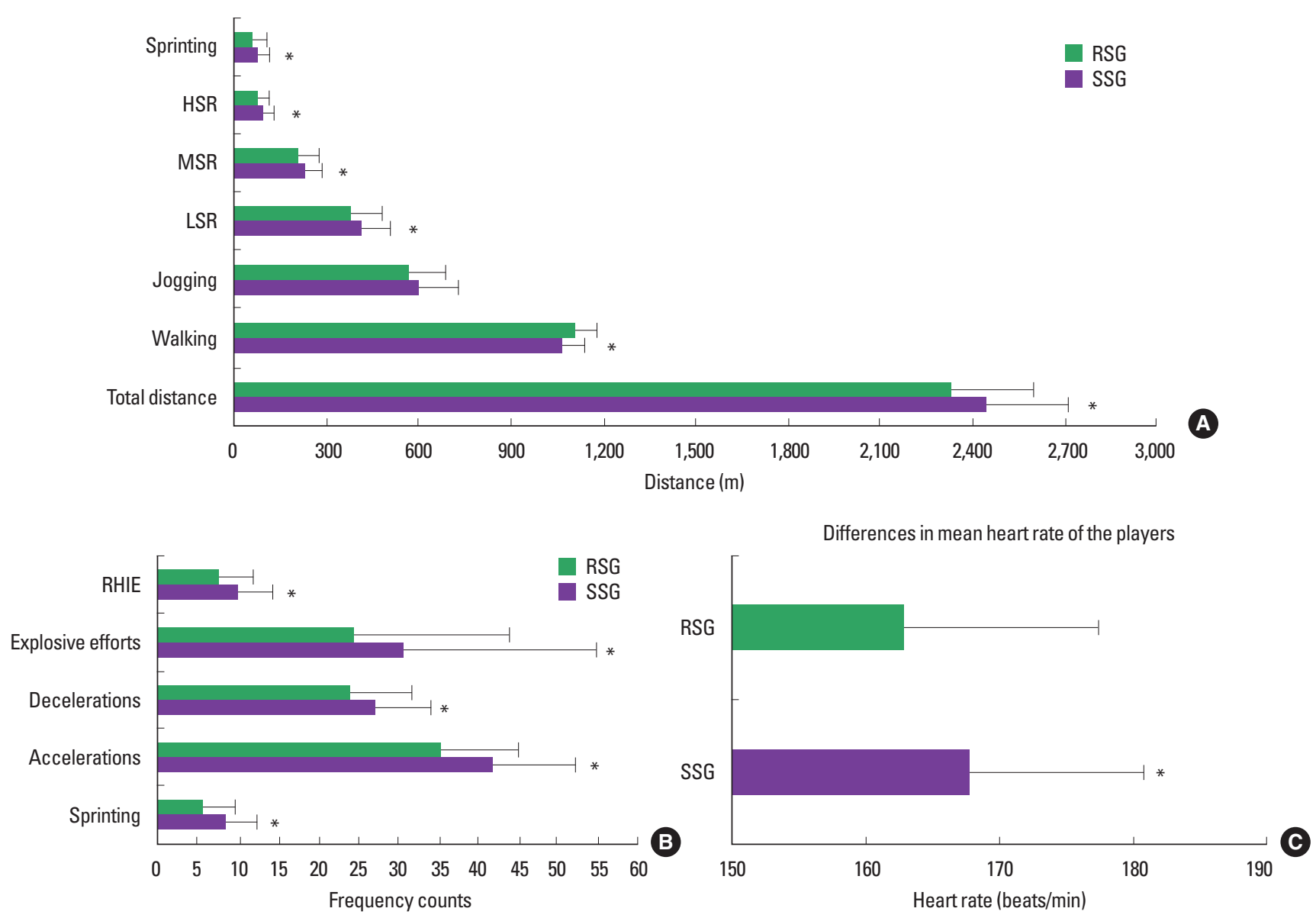

Fig. 2. Comparative analyses of physical demand variables of the players assessed during the SSG and RSG. (A) Differences in activity characteristics of the players. (B) Differences in high intensity activities of the players. The bars express the values calculated in mean \pm standard deviation. Sprinting ( $\geq 18.0 \mathrm{~km} / \mathrm{hr}$ ); HSR, highspeed running (16.0-17.9 km/hr); MSR, moderate-speed running (13.0-15.9 km/hr); LSR, low-speed running (10.0-12.9 km/hr); Jogging (7.0-9.9 km/hr); walking (0$6.9 \mathrm{~km} / \mathrm{hr})$; RHIE, repeated high-intensity efforts; SSG, 8-a-side game with a small pitch dimension; RSG, 11-a-side game with a regular pitch dimension. ${ }^{*} P<0.05$, comparison between SSG and RSG. 


\section{DISCUSSION}

SSG formats (i.e., 7 vs. 7 and 8 vs. 8) have been widely used for official young player games in many soccer-advanced countries, while only 11 vs. 11 format has been applied in official games in Korea. The KFA is considering changing the official game format from RSG to SSG to develop skill performance in young soccer players. Therefore, differences in technical plays and physical aspects between game formats (SSG and RSG) were analyzed for young Korean soccer players. The main findings of this study were that SSG was characterized by more technical plays and greater physical demands than the traditional game format (RSG) for young soccer players.

The players performed a greater number of ball touches and pass variables, apart from long-distance passes, in SSG than in RSG (Fig. 1A). These results are in line with previous studies reporting that SSG allowed more chances for the players to perform ball touches and various passes (Jones and Drust, 2007; Joo et al., 2016). A significantly greater number of passes and successful passes in defense and the attacking $1 / 3$ area as well as forward passes in SSG than in RSG may indicate that SSG has a more offensive play style through build-up from defender to attacker in young soccer players. The reason for these results is likely to be related to increased chances for the players to contact the ball with fewer players during SSG.

There were more shots, all goal shots, valid goal shots, and shots in penalty area in SSG compared to RSG in the present study (Fig. 1B). Joo et al. (2016) also observed a significantly greater number of all goal shots in SSG8 ( 8 vs. 8 on a smaller pitch, $68 \times 47 \mathrm{~m}$ ) than in RSG11 (11 vs. 11 on a regular pitch, $75 \times 47 \mathrm{~m}$ ). However, there was no significant difference in all goal shots and valid goal shots between SSG8 and RSG8 (8 vs. 8 on a regular pitch, $75 \times 47 \mathrm{~m}$ ) in the previous study. Interestingly, the number of all goal shots (1.2 vs. 1.06) and valid goal shots (0.8 vs. 0.34 ) during SSG were higher in the present study compared to the previous study, despite less playing time ( $25 \mathrm{~min}$ vs. $30 \mathrm{~min}$ ). The data from these investigations suggest that the number of all goal shots and valid goal shots seem to be associated with the individual playing area and depth of the pitch for young soccer players. Indeed, the individual playing area $\left(197 \mathrm{~m}^{2}\right.$ vs. $\left.200 \mathrm{~m}^{2}\right)$ and depth of the pitch (62 m vs. $68 \mathrm{~m}$ ) for SSG were smaller in the present study than in the previous study. Because most goals are scored within the penalty area (Wright et al., 2011), the higher number of shots in the penalty area in SSG compared to RSG in the present study suggests that when the pitch dimension is small, young players have increased goal-scoring exposure.

The players completed successful dribbles more often during SSG compared to RSG (Fig. 1C). Similarly, Katis and Kellis (2009) observed a greater number of dribbles during game conditions played with fewer young soccer players on smaller pitch dimensions. The higher number of successful dribbles in SSG compared to RSG may have contributed to wider available space for a player to possess a ball. Indeed, more 1 vs. 1 situation in SSG and 1 vs. 2 situations in RSG were observed in the present study (data not shown). Future research is needed to examine the difference in actual playing area between SSG and RSG for young soccer players. Unlike other technical variables, there were more aerial duels in RSG than in SSG (Fig. 1C). This is supported by a previous study showing that the number of headers was higher in SSG (Katis and Kellis, 2009). These findings suggest that young soccer players cooperate with their teammates via passes on the ground rather than through air balls during SSG. On the other hand, the difference in the number of aerial duels between SSG and RSG can be associated with different team playing formations (Carling, 2011).

To the best of our knowledge, only 2 previous studies have examined the difference in physical demands between SSG and RSG on young soccer players (Capranica et al., 2001; Joo et al., 2016). Significantly greater total distances were covered during jogging, LSR, MSR, HSR, and sprinting in SSG than in RSG (Fig. 2A). The players also completed more high-intensity activities (repeated high-intensity efforts, explosive efforts, decelerations, accelerations, and sprints) during SSG compared to RSG (Fig. 2B). These finding coincide with previous studies reporting that SSG caused increases in physical demands on young soccer players (Joo et al., 2016). A smaller pitch size with fewer players can induce more high-intensity physical activity, enabling performance of technical variables under increased pressure from opponents and coverage of teammate space on defense (Katis and Kellis, 2009; Kelly and Drust, 2009). A significantly higher mean HR was observed in SSG than RSG in the present study. Despite the high mean HR (168 beats/min) during SSG, the values were less than those in a previous study showing that HR exceeded 170 beats $/$ min $84 \%$ of the time during SSG ( 7 vs. 7 on a smaller pitch, $60 \times 40 \mathrm{~m}$ ) and RSG (11 vs. 11 on a regular pitch, $100 \times 65 \mathrm{~m}$ ) in young soccer players (Capranica et al., 2001).

In conclusion, young soccer players performed a greater number of technical variables during SSG than during RSG. Despite greater physical demands during SSG, the exercise intensity seemed to be similar to that reported in previous studies. There- 
fore, the SSG formats applied in the present study can be suitable as official game formats for Korean young soccer players, enabling significantly greater exposure to technical plays without excessive physical demands.

\section{CONFLICT OF INTEREST}

No potential conflict of interest relevant to this article was reported.

\section{REFERENCES}

Abade EA, Gonçalves BV, Silva AM, Leite NM, Castagna C, Sampaio JE. Classifying young soccer players by training performances. Percept Mot Skills 2014;119:971-984.

Abrantes CI, Nunes MI, Maçãs VM, Leite NM, Sampaio JE. Effects of the number of players and game type constraints on heart rate, rating of perceived exertion, and technical actions of small-sided soccer games. J Strength Cond Res 2012;26:976-981.

Aguiar M, Botelho G, Lago C, Maças V, Sampaio J. A review on the effects of soccer small-sided games. J Hum Kinet 2012;33:103-113.

Bangsbo J. Aerobic and anaerobic training in soccer - special emphasis on training of youth players. Copenhagen (Denmark): University of Copenhagen, Institute of Exercise and Sport Sciences; 2007.

Bangsbo J. The physiology of soccer--with special reference to intense intermittent exercise. Acta Physiol Scand Suppl 1994;619:1-155.

Bradley PS, Dellal A, Mohr M, Castellano J, Wilkie A. Gender differences in match performance characteristics of soccer players competing in the UEFA Champions League. Hum Mov Sci 2014;33:159-171.

Capranica L, Tessitore A, Guidetti L, Figura F. Heart rate and match analysis in pre-pubescent soccer players. J Sports Sci 2001;19:379-384.

Carling C. Influence of opposition team formation on physical and skillrelated performance in a professional soccer team. Eur J Sport Sci 2011;
11:155-164.

Casamichana D, Castellano J. Time-motion, heart rate, perceptual and motor behaviour demands in small-sides soccer games: effects of pitch size. J Sports Sci 2010;28:1615-1623.

Castellano J, Casamichana D, Dellal A. Influence of game format and number of players on heart rate responses and physical demands in small-sided soccer games. J Strength Cond Res 2013;27:1295-1303.

Di Salvo V, Baron R, González-Haro C, Gormasz C, Pigozzi F, Bachl N. Sprinting analysis of elite soccer players during European Champions League and UEFA Cup matches. J Sports Sci 2010;28:1489-1494.

Hill-Haas SV, Dawson BT, Coutts AJ, Rowsell GJ. Physiological responses and time-motion characteristics of various small-sided soccer games in youth players. J Sports Sci 2009;27:1-8.

Jones S, Drust B. Physiological and technical demands of $4 \mathrm{v} 4$ and $8 \mathrm{v} 8$ games in elite youth soccer players. Kinesiology 2007;39:150-156.

Joo $\mathrm{CH}$, Hwang-Bo K, Jee $\mathrm{H}$. Technical and physical activities of smallsided games in young Korean soccer players. J Strength Cond Res 2016; 30:2164-2173.

Katis A, Kellis E. Effects of small-sided games on physical conditioning and performance in young soccer players. J Sports Sci Med 2009;8:374380.

Kelly DM, Drust B. The effect of pitch dimensions on heart rate responses and technical demands of small-sided soccer games in elite players. J Sci Med Sport 2009;12:475-479.

Rampinini E, Alberti G, Fiorenza M, Riggio M, Sassi R, Borges TO, Coutts AJ. Accuracy of GPS devices for measuring high-intensity running in field-based team sports. Int J Sports Med 2015;36:49-53.

Reilly T, Bangsbo J, Franks A. Anthropometric and physiological predispositions for elite soccer. J Sports Sci 2000;18:669-683.

Wright C, Atkins S, Polman R, Jones B, Sargeson L. Factors associated with goals and goal scoring opportunities in professional soccer. Int J Perform Anal Sport 2011;11:438-449. 